\title{
$\gamma \mid$ Coffee consumption and death from coronary heart disease in middle aged Norwegian men and women /f
}

\author{
Aage Trverdal, Inger/Stensvold, Kari Solvoll, Olav P/Foss, Per[Lund-Larsen, Kjell/Bjartveit
}

Abstract

Objective-To study the association between number of cups of coffee consumed per day and coronary death when taking other major coronary risk factors into account.

Design-Men and women attending screening and followed up for a mean of 6.4 years.

Setting-Cardiovascular survey performed by ambulatory teams from the National Health Screening Service in Norway.

Participants-All middle aged people in three counties: 19398 men and 19166 women aged 35-54 years who reported neither cardiovascular disease or diabetes nor symptoms of angina pectoris or intermittent claudication.

Main outcome measure-Predictive value of number of cups of coffee consumed per day.

Results - At initial screening total serum cholesterol concentration, high density lipoprotein cholesterol concentration, blood pressure, height, and weight were measured and self reported information about smoking history, physical activity, and coffee drinking habits was recorded. Altogether 168 men and 16 women died of coronary heart disease during follow up. Mean cholesterol concentrations for men and women were almost identical and increased from the lowest to highest coffee consumption group $(13 \cdot 1 \%$ and $10.9 \%$ respectively). With the proportional hazards model and adjustment for age, total serum and high density lipoprotein cholesterol concentrations, systolic blood pressure, and number of cigarettes per day the coefficient for coffee corresponded to a relative risk between nine or more cups of coffee and less than one cup of 2.2 (95\% confidence interval $1 \cdot 1$ to $4 \cdot 5$ ) for men and $5 \cdot 1$ $(0.4$ to 60.3$)$ for women. For men the relative risk varied among the three counties.

Conclusions-Coffee may affect mortality from

National Health Screening Service, Oslo, Norway

Aage Tverdal, CAND REAL, epidemiologist

Inger Stensvold, CAND REAL, research fellow

Per Lund-Larsen, MD, deputy director

Kjell Bjártveit, $\mathrm{MD}$, director

Section for Dietary

Research, Institute for Nutrition Research, University of Oslo, Oslo

Kari Solvoll, nutritionist

Ullevål Hospital, Central Laboratory, Oslo

Olav P Foss, MD, deputy

director

Correspondence to: Dr I Stensvold, National Health Screening Service, PO Box 8155, 0033 Oslo 1 Norway. the incidence of coronary heart disease between high and low coffee consumers in male medical students in a 10 year follow up study ${ }^{4}$; this is much stronger than could be explained by the coffee-cholesterol association. Le Grady et al reported a relative risk of coronary death of 1.7 between consumers of six or more and less than six cups of coffee among white men aged 40-56 after adjustment for serum cholesterol concentration and other variables. ${ }^{5}$ The association between coffee consumption and the other coronary risk factors, such as high density lipoprotein cholesterol concentration, triglyceride concentration, and systolic and diastolic blood pressure, has also been studied cross sectionally, but the direction of the association has been controversial, ${ }^{6-8}$ and the strength of the association has been weaker than that for cholesterol.

In the present study of 38564 men and women aged 35-54 followed for an average of 6.4 years the association between number of cups of coffee per day and death from coronary heart disease is studied when other major coronary risk factors are taken into account.

\section{Methods}

A total of 19398 men and 19166 women aged 35-54 examined during 1977-82 and reporting neither cardiovascular disease or diabetes nor symptoms of angina pectoris or intermittent claudication were followed up to the end of 1986 (mean 6.4 years). This was the second cardiovascular survey in the three counties of Finnmark, Sogn og Fjordane, and Oppland. The same protocol as the first survey three to five years earlier ${ }^{9}$ was adhered to, with the exception of modified criteria for recommending follow up examination. The attendance rate was high $89 \%$ for men and $93 \%$ for women) and was much the same in the three counties.

A questionnaire handed out at the screening to all attenders contained questions about coffee and several other dietary habits. ${ }^{10}$ The question about coffee read: "How many cups of coffee do you usually drink per day" and listed six alternatives from none or less than one cup to nine or more cups of coffee. One cup of coffee is approximately $115 \mathrm{ml}$, with no variation in cup size between the counties, based on informal evidence. ${ }^{10}$ No question about method of brewing coffee was asked. Among the attenders about $90 \%$ returned this questionnaire.

Other variables measured include height, weight, total serum cholesterol concentration, serum triglyceride concentration, serum high density lipoprotein cholesterol concentration, and systolic and diastolic blood pressure, and self reported information about smoking history and physical activity was recorded.

Non-fasting blood samples were drawn and analysed at the Central Laboratory, Ulleval Hospital, Oslo, for serum cholesterol and triglycerides. Serum high density lipoprotein cholesterol concentration was determined at the same laboratory in serum samples from Sogn og Fjordane and Oppland. The serum 
samples from Finnmark were analysed by the laboratory at the University of Tromsø after storage at $-20^{\circ} \mathrm{C}$ for 12 months." The present study used these values for serum high density lipoprotein, adding $0.12 \mathrm{mmol} / \mathrm{l}$ to compensate for storing. "In four municipalities, however, the serum samples were analysed at once, without storage, and these were not included in this study as they were not transferred to our files. Therefore, analyses including serum high density lipoprotein cholesterol show fewer people at risk (about 2500 less) and fewer coronary deaths ( 16 less for men and one less for women).

In 1979, after the screening in Finnmark but before the screening in Sogn og Fjordane and Oppland, the Central Laboratory, Ullevål Hospital, changed to an enzymatic method (with a Technicon AutoAnalyzer) for determining serum cholesterol and triglyceride concentrations. On the basis of an extensive test programme formulas were evolved for expressing the values for samples from Finnmark in terms of the new method. In the present paper all serum lipid values are given in terms of the new method. The Ullevål laboratory has carried out an extensive reference serum programme in order to keep the analytical procedure stable over the years. ${ }^{12}$

Systolic and diastolic blood pressure were measured twice, with a one minute interval, using a sphygmomanometer and standard cuffs. The second measurement was used in this study.

During the follow up period there were 168 deaths from coronary heart disease in men and 16 deaths in women. Sudden death of unknown cause was included among the coronary deaths and constituted 28 of the deaths in men and two of the deaths in women. Coronary death was defined as death coded by Central Bureau of Statistics as 410-411, 412.0-412.3, 413, 782.4 , or 795 in the Norwegian version of the eighth revision of the International Classification of Diseases. ${ }^{13}$

The number of observation years was calculated for each person from the time of examination to the time of death, time of emigration, or 31 December 1986, in this priority. Age adjustments were done by the indirect method, using all data in one year age groups as the standard population. Adjustment for both age with another variable was done in the same way, with the other variable appropriately categorised.

Trend was tested by modelling $\ln (\mathrm{O} / \mathrm{E})$ as a linear function of number of cups of coffee, where $\mathrm{O}$ is the observed and $\mathrm{E}$ is the expected number of deaths. This was done with generalised linear interactive modelling, assuming $\mathrm{O}$ to have a Poisson distribution. ${ }^{14-16}$ Coffee consumption groups were coded as $1,2,3,4,5,6$.

Cox proportional hazards regressions with appropriate covariates were also run (biomedical data programs package $2 \mathrm{~L}$ ), ${ }^{17}$ with coffee consumption groups coded as above. The relative risk between nine cups or more and less than one cup (adjusted for the other covariates) was the exponential of the estimated coffee coefficient times five.

\section{Results}

Mean cholesterol concentration increased by $0.76 \mathrm{mmol} / \mathrm{l}$ from lowest to highest coffee consumption group in men $(13 \cdot 1 \%)$ and $0.63 \mathrm{mmol} / \mathrm{l}$ in women $(10 \cdot 9 \%)$ (table I). The mean concentrations for men and women were almost identical in each of the coffee consumption groups, except in the group having nine cups or more, in which the mean was higher in men. In this study population the age specific mean was higher in men than women at ages 35-39 and 40-44, equal at ages 45-49, and lower at ages 50-54. ${ }^{1 *}$

The age adjusted mortality rose steadily in men consuming more than two cups. The two lowest consumption groups had almost the same rate and few deaths (table I). When adjustment was also made for number of cigarettes, which was related to coffee consumption (product moment correlation coefficient 0.35 for men and 0.33 for women), the trend became weaker but remained significant. Adjustment for cholesterol concentration in addition to age also reduced the linear trend, but the relation remained significant. This suggests that there is an effect of coffee consumption on mortality from coronary heart disease over and above the effect on serum cholesterol concentration.

Among women there were few deaths, and an excess risk was found only for the two highest consumption

TABLE I-Coffee consumption, cholesterol concentration, and deaths from coronary heart disease in Norwegians aged 35-54

\begin{tabular}{|c|c|c|c|c|c|c|c|}
\hline & \multicolumn{6}{|c|}{ No of cups of coffee } & \multirow{2}{*}{$\begin{array}{c}\text { Linear trend } \\
\text { coefficient } \\
(95 \% \\
\text { confidence interval })\end{array}$} \\
\hline & $<1$ & $1-2$ & $3-4$ & $5-6$ & $7-8$ & $\geqslant 9$ & \\
\hline \multicolumn{8}{|c|}{ Men } \\
\hline No at risk & 870 & 1651 & 4995 & 5845 & 3481 & 2556 & \\
\hline Mean cholesterol (adjusted for age $(\mathrm{mmol} / \mathrm{l})$ & $5 \cdot 80$ & 5.96 & $6 \cdot 15$ & $6 \cdot 25$ & $6 \cdot 37$ & 6.56 & \\
\hline No of deaths & 3 & 6 & 29 & 45 & 42 & 43 & \\
\hline \multicolumn{8}{|l|}{ Deaths per 100000 observed years adjusted for: } \\
\hline Age & 62 & 61 & 92 & 119 & 186 & 244 & $0.33(0.21$ to 0.45$)$ \\
\hline Age and No of cigarettes per day & 100 & 83 & 111 & 121 & 158 & 179 & $0.17(0.05$ to 0.29$)$ \\
\hline Age and serum cholesterol & 81 & 73 & 96 & 121 & 177 & 203 & $0.25(0.13$ to 0.37$)$ \\
\hline \multicolumn{8}{|c|}{ Women } \\
\hline No at risk & 593 & 1771 & 6656 & 6164 & 2629 & 1353 & \\
\hline Mean cholesterol (adjusted for age) $(\mathrm{mmol} / \mathrm{l})$ & $5 \cdot 80$ & 6.01 & $6 \cdot 14$ & $6 \cdot 25$ & $6 \cdot 34$ & $6 \cdot 43$ & \\
\hline \multirow{2}{*}{\multicolumn{8}{|c|}{ Deaths per 100000 observed years, adjusted for: }} \\
\hline & & & & & & & \\
\hline Age & & 18 & 2 & 8 & 24 & 69 & $0.79(0.34$ to 1.24$)$ \\
\hline Age and No of cigarettes per day & & 24 & 3 & 7 & 21 & 50 & $0.45(0.01$ to 0.89$)$ \\
\hline Age and serum cholesterol & & 24 & 3 & 7 & 21 & 50 & $0.49(0.05$ to 0.93$)$ \\
\hline
\end{tabular}

TABLE II - Coefficients (95\% confidence intervals) estimated by Cox proportional hazards regressions with coronary death as end point in Norwegians aged 35-54. (Men at risk=18039, women=18065; deaths in men $=152$, women $=15$ )

\begin{tabular}{|c|c|c|}
\hline & Men & Women \\
\hline Age (years) & $0.073 \quad(0.040$ to 0.106$)$ & $0.063(-0.050$ to 0.176$)$ \\
\hline Systolic blood pressure $(\mathrm{mm} \mathrm{Hg})$ & $(0.014$ to 0.032$)$ & $0.029 \quad(0.011$ to 0.047$)$ \\
\hline No of cigarettes per day & $(0.020$ to 0.053$)$ & $0.095 \quad(0.040$ to $0 \cdot 149)$ \\
\hline Total cholesterol $(\mathrm{mmol} / \mathrm{l})$ & $0.337 \quad(0.210$ to 0.464$)$ & $0.441 \quad(0.177$ to 0.705$)$ \\
\hline High density lipoprotein cholesterol $(\mathrm{mmol} / \mathrm{l})$ & $-0.671(-1.209$ to -0.133$)$ & $-0.832(-2.373$ to 0.708$)$ \\
\hline Coffee (coded as $1,2,3,4,5,6)$ & $0.159 \quad(0.017$ to 0.301$)$ & $0.327(-0.166$ to 0.820$)$ \\
\hline
\end{tabular}

groups (table I). The relation was, however, stronger than for men, but a comparison of the trend coefficients between men and women did not show a significant difference. Adjustment for number of cigarettes or alternatively for serum cholesterol concentration in addition to age gave a considerably weaker relation, but the coefficient remained significant in both cases.

Table II shows the coefficients estimated with the Cox proportional hazards regression. For men this corresponded to a relative risk pf $2 \cdot 2$ between nine or 
more cups of coffee and less than one cup. An increase of two cups of coffee was associated with the same relative risk as an increase of 4.3 cigarettes, $6.9 \mathrm{~mm} \mathrm{Hg}$ systolic blood pressure, or $0.47 \mathrm{mmol} / 1$ total cholesterol or a decrease of $0.24 \mathrm{mmol} / \mathrm{l}$ high density lipoprotein cholesterol.

For women the coffee coefficient was not significant, although it was larger than for men. The effect of increasing coffee consumption by two cups is equalled by 3.5 cigarettes, $11.2 \mathrm{~mm} \mathrm{Hg}$ systolic blood pressure, or $0.74 \mathrm{mmol} / \mathrm{l}$ total cholesterol or a decrease of $0.39 \mathrm{mmol} / \mathrm{l}$ serum high density lipoprotein cholesterol.

Table III shows relative risks between nine or more cups of coffee and less than one cup in certain subgroups, estimated from Cox proportional hazards models and adjusted successively for an increasing number of covariates. Because there were few deaths in women these analyses have been done only for men.

Among the three counties, Oppland showed the strongest relation of coffee to death from coronary heart disease. In the other counties the relation was not significant. Testing a common coefficient in the three counties in men aged $40-54$ gave borderline significance $(\mathrm{p}=0 \cdot 05)$.

A mortality follow up of a similar population that was screened during 1973-6 showed that among seven risk factors relating to stress only "being on disability pension" was an independent predictor of coronary death. ${ }^{19}$ County specific Cox regressions with disability pension $(0=$ no, $1=$ yes $)$, body mass index, and physical activity during leisure (coded 1, 2, 3, or 4 with increasing activity) in addition to age, systolic blood pressure, number of cigarettes, high density lipoprotein concentration, and total cholesterol concentration gave relative risks ( $\geqslant 9$ cups $v<1$ cup) of $6.9(95 \%$ confidence interval $2 \cdot 1$ to $22 \cdot 6)$ for Oppland, $1 \cdot 3(0 \cdot 2$ to 6.9) for Sogn og Fiordane, and $0.9(0.3$ to 2.8$)$ for Finnmark. When these are compared with the data in table III it can be seen that adding these three variables had only a minor impact on the relation of coffee to death from coronary heart disease.

An interaction term between cups of coffee and high density lipoprotein cholesterol concentration, in addition to the main effects and age and total serum cholesterol concentration, was not significant $(p=0 \cdot 15)$, nor was an interaction term between cups of coffee and systolic blood pressure $(p=0 \cdot 44)$. Nevertheless, it mav be noted that the relation of coffee to death from coronary heart disease was strongest in the supposed high risk part of the range of high density lipoprotein cholesterol concentrations and in the low risk part of the range of systolic blood pressures.

\section{Discussion}

We found that coffee intake was significantly related to death from coronary heart disease in men, and also when other major risk factors were taken into consideration. In women coffee intake was also related to death from coronary heart disease. The estimated coefficients were generally larger for women than for men, but when all major risk factors were taken into account the coffee gradient was not significant.

Over $80 \%$ of those screened responded to the question on coffee. Comparison of the age adjusted mortality from coronary heart disease for those who did and did not answer the question gave a ratio of $1 \cdot 17$ for men and 1.25 for women. Thus, response bias could explain only a small part of the relation between coffee intake and death from coronary heart disease.

Because the information on coffee was collected from a self administered questionnaire there is a possibility of misclassification. A comparison with 24 hour recall of a sample in seven municipalities in the first cardiovascular survey (three to five years earlier) of

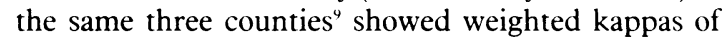
0.36 for men and 0.29 for women. ${ }^{21}$ As the information about coffee hardly influenced the diagnostic procedure, the misclassification of consumption in this study means, if anything, that the association between coffee consumption and death from coronary heart disease has been underestimated.

On the other hand, it is difficult to assess the implication of adjusting for an imperfect estimate of a person's usual cholesterol concentration or blood pressure obtained on a single occasion. Studies that use a more representative estimate of the variables, such as the mean of two measurements taken a few weeks or months apart, will give an even better basis for assessing the role of coffee dose as an independent predictor of death from coronary heart disease.

The method of coffee brewing was not asked for, but a survey of the three counties in 1985-7 showed that in Finnmark $80 \%$ used boiled coffee. In Oppland $55 \%$ and in Sogn og Fjordane $80 \%$ used filter coffee (I Stensvold, unpublished data). Cross sectional studies in Norway have shown that the coffee-cholesterol association is strongest for boiled coffee. ${ }^{26}$

Oppland county, where the association was strongest, was surveyed last and thus had the shortest

TABLE III-Relative risks (95\% confidence intervals) for nine cups or more of coffee compared with less than one cup per day for death from coronary heart disease in Norwegian men aged 35-54

\begin{tabular}{|c|c|c|c|c|}
\hline & \multirow[b]{2}{*}{$\begin{array}{c}\text { No } \\
\text { of } \\
\text { deathst }\end{array}$} & \multicolumn{3}{|c|}{ Relative risks ${ }^{\star}$ adjusted for: } \\
\hline & & Age & $\begin{array}{c}\text { Age, } \\
\text { serum } \\
\text { cholesterol }\end{array}$ & $\begin{array}{l}\text { Age, high density lipoprotein and total } \\
\text { cholesterol, systolic blood pressure, } \\
\text { No of cigarettes/day }\end{array}$ \\
\hline \multicolumn{5}{|l|}{ Counties: } \\
\hline Oppland & 58 & $13 \cdot 3(4 \cdot 4$ to $40 \cdot 0)$ & $11 \cdot 5(3 \cdot 8$ to $35 \cdot 1)$ & $7 \cdot 3(2 \cdot 2$ to $23 \cdot 8)$ \\
\hline Sogn og Fiordane & 31 & $3 \cdot 2(0 \cdot 7$ to $13 \cdot 5)$ & $2 \cdot 9(0 \cdot 7$ to $12 \cdot 4)$ & $1.4(0 \cdot 3$ to $6 \cdot 5)$ \\
\hline Finnmark & 79,63 & $1.9(0.7$ to $4 \cdot 8)$ & $1 \cdot 6(0 \cdot 6$ to $4 \cdot 0)$ & $1.0(0.3$ to $2 \cdot 9)$ \\
\hline \multicolumn{5}{|l|}{ Cigarette smoking: } \\
\hline Never smoked & 14,13 & $3 \cdot 3(0 \cdot 4$ to $28 \cdot 5)$ & $2 \cdot 6(0 \cdot 3$ to $23 \cdot 1)$ & $3 \cdot 6(0.3$ to $37 \cdot 4)$ 屯: \\
\hline Ex-smokers & 22,21 & $0 \cdot 9(0 \cdot 2$ to $5 \cdot 4)$ & $0 \cdot 7(0 \cdot 1$ to $4 \cdot 3)$ & $0 \cdot 6(0 \cdot 1$ to $4 \cdot 1) f$ \\
\hline Current daily smokers & 132,118 & $2 \cdot 3(1 \cdot 1$ to $4 \cdot 9)$ & $1.9(0.9$ to $4 \cdot 1)$ & $2 \cdot 1(0.9+0.7)$ \\
\hline \multicolumn{5}{|l|}{ Sugar in coffec: } \\
\hline No & 63,59 & $6 \cdot 6(2 \cdot 4$ to $18 \cdot 5)$ & $5.4(1.9$ to $15 \cdot 2)$ & $4 \cdot 1(1 \cdot 3 t 013 \cdot 2)$ \\
\hline Yes & 100,88 & $3 \cdot 3(1 \cdot 4$ to $7 \cdot 7)$ & $2 \cdot 6(1 \cdot 1$ to $6 \cdot 2)$ & $1 \cdot 6(0 \cdot 6104 \cdot 3)$ \\
\hline \multicolumn{5}{|c|}{ High density lipoprotein cholesterol $(\mathrm{mmol} / \mathrm{l})$ : } \\
\hline$<1 \cdot 2$ & 89 & $8 \cdot 2(3 \cdot 3$ to $20 \cdot 2)$ & $6 \cdot 1(2 \cdot 4$ to $15 \cdot 1)$ & $4 \cdot 6(1.7$ to $12 \cdot 1) \mathrm{s}$ \\
\hline$\geqslant 1 \cdot 2$ & 63 & $1 \cdot 7(0 \cdot 6$ to $4 \cdot 6)$ & $1.4(0.5$ to 3.9$)$ & $0.9(0.3$ to $2 \cdot 6) 5$ \\
\hline \multicolumn{5}{|c|}{ Systolic blood pressure $(\mathrm{mm} \mathrm{Hg})$ : } \\
\hline$<1+0$ & 84,79 & $6 \cdot 4(2 \cdot 6$ to $15 \cdot 8)$ & $4.7(1.9$ to 11.7$)$ & $3 \cdot 3(1 \cdot 2$ to $9 \cdot 0)$ \\
\hline$\geqslant 140$ & 84,73 & $2 \cdot 7(1 \cdot 1$ to $6 \cdot 7)$ & $2 \cdot 4(1 \cdot 0$ to $5 \cdot 9)$ & $1.3(0.5$ to 3.5$)$ \\
\hline \multicolumn{5}{|c|}{ Total serum cholesterol $(\mathrm{mmol} / \mathrm{l})$ : } \\
\hline$<6 \cdot 2$ & 44,40 & $3 \cdot 6 \cdot 1 \cdot 1$ to $11 \cdot 9)$ & & $2 \cdot 8(0.7$ to $10 \cdot 5) 9$ \\
\hline$\geqslant 6 \cdot 2$ & 124,112 & $3 \cdot 5(1 \cdot 7$ to $7 \cdot 2)$ & & $2 \cdot 3(1 \cdot 0$ to $5 \cdot 2)$ \\
\hline
\end{tabular}

^Estimated by Cox proportional hazards regressions.

†Both maximum and minimum are given when numbers differ with number of covariates.

¡Not adjusted for number of cigarettes.

Not adjusted for high density lipoprotein cholesterol. Not adjusted for systolic blood pressure.

Not adjusted for serum cholesterol. 
follow up period. Finnmark, where the association was weakest, had the longest follow up time. This inverse relationship between the length of follow up and the strength of the effect of coffee on coronary death fits in with the finding of La Croix et al,${ }^{4}$ who measured coffee intake several times and found that the estimate of relative risk based on the most recent measurement or on a cumulative measure was greater than that based on the first measurement of consumption.

Subjects who had a high risk were recommended to have a further examination and intervention, if appropriate. For men the percentage referred increased with increasing coffee consumption, whereas for women it by and large decreased. Accordingly, the intervention may have weakened the relation between coffee consumption and death from coronary heart disease in men and strengthened it in women.

The major uncertainty relating to previous studies has been whether the apparent relation between coffee and death from coronary heart disease is mediated through correlated lifestyle factors. ${ }^{21}$ We took physical activity and stress into consideration, but we have no information about socioeconomic status. As regards diet, a cross sectional study of the first Oppland survey carried out five years before the Oppland part of this study showed that dietary habits and lifestyle could not explain the association of coffee and serum cholesterol concentration, ${ }^{10}$ and a follow up relating dietary habits and lifestyle to death from coronary heart disease has not yet been done.

We conclude that coffee consumption is fairly strongly related to death from coronary heart disease, but a potential causal role is weakened by the varying strength of the relation in different groups. To verify the observed effect of coffee on deaths from coronary heart disease over and above the effect on cholesterol concentration, cholesterol concentration must be determined more accurately than by a single measurement.
1 Thelle DS, Heyden S, Fodor JG. Coffee and cholesterol in epidemiological and experimental studies. Atherosclerosis 1987;67:97-103

Bonaa K, Arnesen E, Thelle DS, Forde OH. Coffee and cholesterol: is it all in the brewing? The Tromso study. Br Med f 1988;297:1103-4.

3 Tverdal A. Systolic and diastolic blood pressures as predictors of coronary heart disease in middle aged Norwegian men. Br Med f 1987;294:671-3.

4 La Croix AZ, Mead LA, Liang K-Y, Thomas CB, Pearson TA. Coffee consumption and the incidence of coronary heart disease. $N$ Engl $f$ Med 1986;315:977-82.

5 Le Grady D, Dyer AR, Shekelle RB, et al. Coffee consumption and mortality in the Chicago Western Electric Company study. Am F Epidemiol 1987;126: $803-12$.

6 Stensvold I, Tverdal A, Foss OP. The effect of coffee on blood lipids and blood pressure. Results from a Norwegian cross-sectional study, men and women, 10-42 years. $f$ Clin Epidemiol 1989;42:877-84.

7 Williams PT, Wood PD, Vranizan KM, Albers JJ, Garay SC, Taylor CB. Coffee intake and elevated cholesterol and apolipoprotein B levels in men. FAMA 1985;253:1407-11

8 Periti M, Salvaggio A, Quaglia G, Di Marzio L. Coffee consumption and blood pressure: an Italian study. Clin Sci 1987;72:443-7.

9 Bjartveit K, Foss OP, Gjervig T, Lund-Larsen PG. The cardiovascular disease study in Norwegian counties: background and organization. Acta Med Scand 1979; Suppl 634:1-70.

10 Solvoll K, Selmer R, Loken EB, Foss OP, Trygg K. Coffee, dietary habits and serum cholesterol among men and women 35-49 years of age. Am f Epidemiol 1989;129:1277-88.

11 Thelle DS, Forde OH, Arnesen E. Distribution of high-density lipoprotein cholesterol according to age, sex, and ethnic origin: cardiovascular disease study in Finnmark 1977. J Epidemiol Community Health 1982;36:243-7.

12 Tretli S, Bjartveit K, Foss OP, Haider T, Lund-Larsen PG. Intervention on cardiovascular disease risk factors in Finnmark county: changes after a cardiovascular disease risk factors in Finnmark c3.

13 World Health Organisation. International classification of diseases, injuries, and World Health Organisation. International classification of diseases, injurios
causes of death. Eighth revision. Oslo: Statistisk Sentralbyrá, 1973.

14 Healy MJR. GLIM: an introduction. Oxford: Oxford University Press, 1988.

15 Payne CD, ed. The GLIM system, release 3.77, revision a. Oxford: Numerical Algorithms Group, 1986.

16 Berry G. The analysis of mortality by the subject-years method. Biometric 1983;39:173-84.

17 Dixon WJ, ed. BMDP statistical software. Berkeley: University of California Press, 1981.

18 National Health Screening Service, Health Services of Finnmark, Sogn og Fjordane and Oppland counties, Ullevål Hospital, Central Laboratory, Oslo. The cardiovascular disease study in Norwegian counties. Results from the second screening. Oslo: National Health Screening Service, 1988.

19 Tverdal A. A mortality follow-up of persons invited to a cardiovascular disease study in five areas in Norvay. Oslo: National Health Screening Service, 1989.

20 Solvoll $\mathrm{K}$. Comparison of dietary data from self administered questionnaire and 24-hour recall. Oslo: Avdeling for Kostholdsforskning, Institutt for Ernæringsforskning, 1983.

21 Vandongen R. Coffee and heart disease: the hypothesis re-examined. Med J Aust 1988;148:161-2.

\title{
Blood pressure response to percutaneous transluminal angioplasty for renovascular hypertension: an overview of published series
}

\author{
Lawrence E Ramsay, Patrick C Waller
}

\section{Abstract}

Objective-To evaluate the efficacy and safety of transluminal angioplasty in the treatment of renovascular hypertension.

Design-Overview of 10 published case series.

Setting-Mostly tertiary referral centres.

Patients - 691 Patients treated by angioplasty for renovascular hypertension, 464 for atherosclerotic stenosis, 193 for fibromuscular stenosis, and the remainder mainly for post-transplant stenosis. The inclusion criteria varied considerably between studies.

Interventions-Percutaneous transluminal renal artery angioplasty (successful or attempted); prophylaxis against restenosis in most cases. Policies on antihypertensive drug treatment and follow up varied.

Main outcome measures-Blood pressure response as defined in the individual papers, with each patient classified as either cured, improved, or failed. Each study used a different definition of improvement in blood pressure. Complication rates.

Results-Follow up data were available for 670 patients. The procedure was technically successful in 611 patients $(88 \%)$. The overall cure rate for hypertension was $24 \%$ ( 163 patients) and a further 43\% (286) were "improved." There were large and significant variations between studies in the rate of technical success and also the blood pressure outcome. In each series patients with fibromuscular lesions, had a higher cure rate than those with atherosclerotic lesions (overall cure $50 \%$ v $19 \%$ odds ratio $3.9,95 \%$ confidence interval 2.6 to 6.0 ). Complications were reported in $63(9 \%)$ patients and three patients died $(0 \cdot 4 \%)$.

Conclusions - The evidence for the value of angioplasty in treating renovascular hypertension has serious limitations. Angioplasty for fibromuscular disease seems to be worth while, but for atherosclerotic lesions the benefit appears to be small and its efficacy needs to be compared with medical therapy in randomised trials. These findings emphasise the importance of early formal evaluation of new therapeutic techniques.

\section{Introduction}

During the past 10 years percutaneous transluminal angioplasty has been used widely to treat renovascular hypertension, and several case series have been pub- 